\title{
Geleitwort Anne Lévy
}

In der Schweiz können die Patientinnen und Patienten mit einer sicheren und qualitativ hochstehenden Behandlung rechnen. Doch auch hier kommt es bei fast 10 Prozent der Patientinnen und Patienten, die in ein Spital eintreten, zu einem unerwünschten medizinischen Zwischenfall. Dazu gehören Komplikationen während einer Operation, eine falsche Medikation oder eine Infektion. Vermutlich könnte die Hälfte dieser Fälle vermieden werden. Es werden hierzulande bereits viele Anstrengungen unternommen.

Mit der Implementierung von Patientensicherheitsmanagementsystemen und des Konzepts der Sicherheitskultur auf der Ebene der Leistungserbringung werden wichtige Voraussetzungen zur Verbesserung der Patientensicherheit geschaffen. Das vorliegende Buch bietet hierzu einen praxisorientierten Überblick und wertvolle Unterstützung für die konkrete Umsetzung in die Alltagspraxis.

Das reicht jedoch nicht aus, um eine hohe Patientensicherheit aufrechtzuerhalten und kontinuierlich zu verbessern. Sie wird in der Schweiz als eine zentrale Dimension der Qualität verstanden. So wird die Wirksamkeit des Patientensicherheitsmanagements gestärkt durch die Institutionalisierung der Qualitätsentwicklung über die betriebliche Ebene hinaus. Die Akteure orientieren sich dabei an einem Regelkreissystem. Schwächen sollen systematisch erkannt werden. Auf Basis von Daten soll entschieden werden, in welchen inhaltlichen Themenfeldern Verbesserungen notwendig sind. Vorbildliche Methoden oder Vorgehensweisen sollen verbreitet und zur neuen Alltagspraxis werden.

Es zeigt sich eindrücklich: Patientensicherheitsmanagement ist kein Selbstzweck! Es geht um die systematische Gewährleistung und kontinuierliche Verbesserung des Niveaus der Qualität und Patientensicherheit beim Einsatz der Ressourcen. Patientensicherheitsmanagement stellt auf allen Ebenen des Gesundheitswesens Prozesse, Leadership und das Engagement sicher. Unser aller Engagement ist notwendig, damit Risiken für die Patientinnen und Patienten reduziert und Verbesserungen implementiert werden.

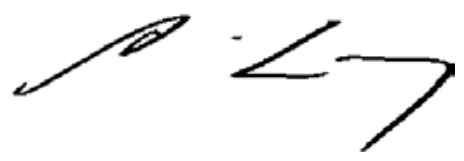

Anne Lévy

Direktorin Bundesamt für Gesundheit BAG 
